\title{
Anomalous structural evolution of soft particles: Equibrium liquid state theory
}

\author{
Hugo Jacquin ${ }^{1}$ and Ludovic Berthier ${ }^{2}$ \\ ${ }^{1}$ Laboratoire Matière et Systèmes Complexes, UMR CNRS 705\%, Université Paris Diderot - Paris \%, \\ 10 rue Alice Domon et Léonie Duquet, 75205 Paris cedex 13, France \\ ${ }^{2}$ Laboratoire des Colloïdes, Verres et Nanomatériaux, \\ Université Montpellier II and CNRS, 34095 Montpellier, France
}

(Dated: November 9, 2018)

\begin{abstract}
We use the hyper-netted chain approximation of liquid state theory to analyze the evolution with density of the pair correlation function in a model of soft spheres with harmonic repulsion. As observed in recent experiments on jammed soft particles, theory predicts an 'anomalous' (nonmonotonic) evolution of the intensity of the first peak when density is increased at constant temperature. This structural anomaly is a direct consequence of particle softness, and can be explained from purely equilibrium considerations, emphasizing the generality of the phenomenon. This anomaly is also predicted to have a non-trivial, ' $\mathcal{S}$-shaped', evolution with temperature, as a result of a competition between three distinct effects, which we describe in detail. Computer simulations support our predictions.
\end{abstract}

PACS numbers: 05.20.Jj, 64.70.qd, 83.80.Fg

In very recent work $[1,2,3,4]$, the structure of soft repulsive particles was analyzed experimentally and numerically. Particular attention was paid to a nonmonotonic behaviour of the first peak of the pair correlation with increasing density, as shown in Fig. 1 In the limit where particles are infinitely hard, the height of the first peak diverges when approaching a maximal packing fraction where the pressure diverges [5, 6], and configurations with larger density cannot be explored. Thus, the nonmonotonic height of the pair correlation function $g(r)$ was understood as a smooth, finite-temperature, signature of this jamming transition [1]. While simulations study soft repulsive potentials at finite temperatures [1, 4], experiments introduce instead particle softness, which similarly allows for finite overlaps between particles. For instance, in the very elegant experiment of Ref. [3], the pair correlation function of a slowly swelling assembly of (compressible) tapioca pearls is followed, see Fig. 1.

These studies were performed in nonequilibrium conditions following a preparation protocol which varies from one experiment to another. Here, 'nonequilibrium' means that particles rearrangements are rare during the course of an experiment, and do not allow the system to sample equilibrium trajectories at any given state point, even at finite temperature. The jamming transition cannot be probed at thermal equilibrium, because the system first hits a glass transition and cannot maintain ergodic behaviour at such large densities 7, 8, 9]. Thus, the structural evolution along particular nonequilibrium trajectories does not result from ensemble averages in the usual sense. When comparing two different state points, it is understood that a single configuration is transported from one state point to another along a specific path. This experimental procedure suggests that sample preparation protocols might well play an important role.

There are several questions left unanswered by these studies. First, the origin of the maximum is systematically attributed to an underlying $T=0$ jamming transition, and it is thus unclear how the phenomenon should be described theoretically. Second, the effects of sample preparation were either not discussed, or claimed to be negligible [1]. This seems to contradict recent reports that the location of the jamming transition is sensitively protocol dependent 10, 11, 12, 13] a conclusion which should extend to finite temperatures as well. Third, the location of the maximum was predicted to obey a simple scaling behaviour near zero temperature [1] , as confirmed by simulations spanning a large temperature window [4]. However, this prediction must break down when temperature is large enough to allow relaxation in the system, but this has not been explored.

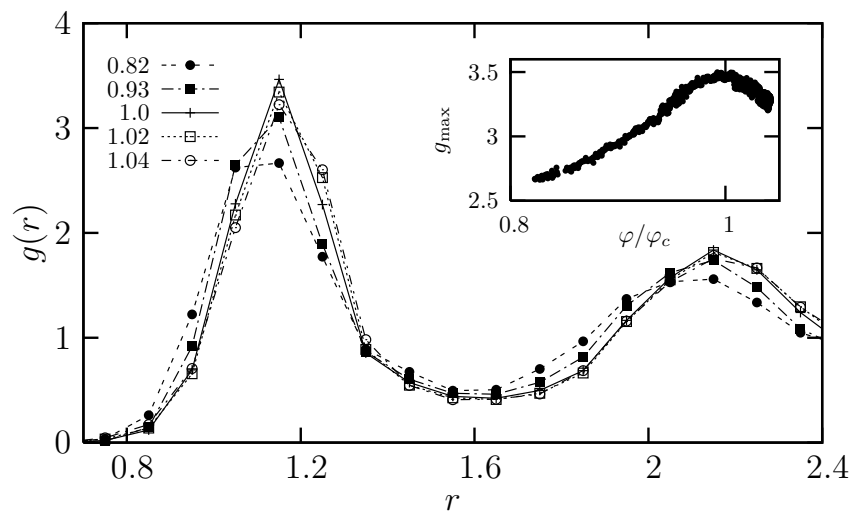

FIG. 1: Nonmonotonic evolution with increasing density of the height of the first peak in the pair correlation of a slowly swelling assembly of (soft) tapioca pearls. Volume fractions $\varphi$ are normalized with respect to that of the maximum location at $\varphi_{c}$. The inset shows the height of the first peak near $r \approx 1.1$ as a function of $\varphi / \varphi_{c}$. These data are adapted from Ref. [3], and were kindly provided by $\mathrm{X}$. Cheng. 
In this paper, our aim is to gain theoretical insight into the behaviour of the pair correlation function of dense assemblies of soft repulsive particles and to address the three above questions. We suggest that the simplest starting point is equilibrium statistical mechanics, and we use the tools of liquid state theory to attack the problem. Although we know beforehand that our results should eventually become inapplicable to the low temperature regime where simulations and experiments fail to probe equilibrium configurations, our simple approach provides useful insights, which we report in this short note.

We follow Refs. [1, 4] and study an assembly of $N$ soft repulsive particles interacting through harmonic repulsion:

$$
V\left(r_{i j}<1\right)=\epsilon\left(1-r_{i j}\right)^{2}
$$

where $r_{i j}=\left|r_{i}-r_{j}\right|$ denotes the distance between particles $i$ and $j$. Particles separated by $r_{i j}>1$ do not interact, $V\left(r_{i j}>1\right)=0$. In the following, we report temperatures in units of $\epsilon$, and the particle diameter sets the unit lengthscale. We study the pair correlation function defined by

$$
g(r)=\frac{1}{\rho N} \sum_{i \neq j}\left\langle\delta\left(r-r_{i j}\right)\right\rangle,
$$

where the brackets indicate an ensemble average at thermal equilibrium, and $\rho=N / V$ is the number density. We use the hyper-netted chain (HNC) closure relation for the pair correlation function [14],

$$
g(r)=\exp [-\beta V(r)+g(r)-1-c(r)]
$$

for the pair potential $V(r)$ in Eq. (11). We have defined $\beta=1 / T$, where $T$ is the temperature. In Eq. (3), $c(r)$ is the direct correlation function defined through the Ornstein-Zernike equation:

$$
g(r)-1=c(r)+\rho \int d r^{\prime} c\left(r-r^{\prime}\right)\left(g\left(r^{\prime}\right)-1\right) .
$$

Although $\mathrm{HNC}$ is known to become quantitatively inaccurate in the hard sphere limit at the large densities investigated here [14], it is a convenient tool to study the interplay between temperature and dynamics in soft repulsive spheres. For the relatively large temperatures studied below, we shall find that $\mathrm{HNC}$ in fact compares quite well with simulations, at least qualitatively.

We solve the HNC closure relation in Eq. (3) numerically. To this end, we discretize $g(r)$ between $r=0$ and $r=32$, using $2^{15}$ points and use a standard Picard iterative procedure, as described in more details in Ref. 15]. As a result, we obtain $g(r)$ over a broad range of temperatures, $T \in[0.00076,0.5]$ and volume fractions, $\varphi=\pi \rho / 6 \in[0.5,1.2]$.

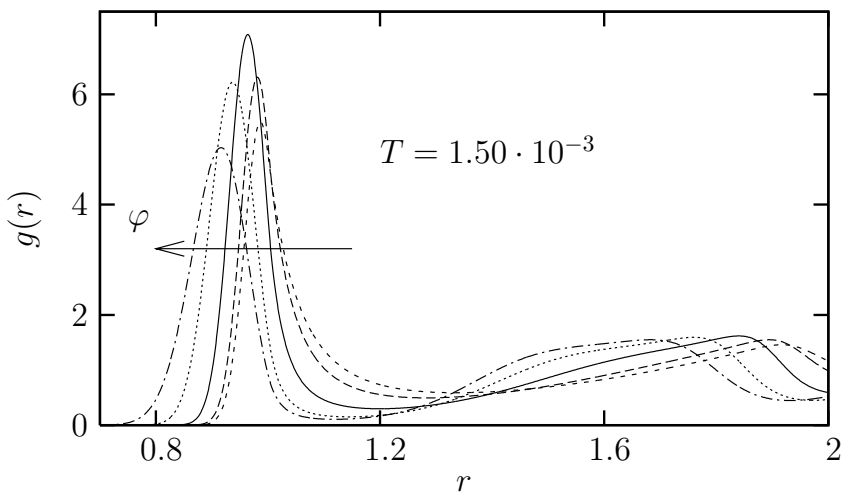

FIG. 2: Nonmonotonic evolution of the height of the first peak in the pair correlation $g(r)$, obtained using the hyper-netted chain approximation. The temperature $T$ is fixed, and the volume fraction increases from right to left with $\varphi=0.600$, $0.663,0.777,0.900$, and 1.00 .

Our first result is shown in Fig. 2, where the pair correlation of harmonic spheres at constant temperature, $T=1.5 \cdot 10^{-3}$, is shown for volume fractions increasing from $\varphi=0.60$ up to $\varphi=1.0$. While the position of the first peak shifts to smaller distances as a result of the compression, the height of the first peak displays a nonmonotonic evolution. For this temperature, it increases up to $\varphi \approx 0.777$, and then decreases for larger volume fractions. This evolution is very similar to the one reported in Fig. 1. In Fig. 2 it can be seen that the second peak of $g(r)$ also displays a nonmonotonic behaviour, while we find the subsequent peaks do not. Remarkably, the same observations were reported in experiments [3].

In the context of jamming and glassy physics, this result is unexpected, since in simple glass-forming liquids, modelled for instance as Lennard-Jones fluids, the first peak of $g(r)$ typically increases with density at large density, contrary to the system of harmonic spheres, where it instead decreases, see Fig. 2

This result shows that a nonmonotonic structural evolution of $g(r)$ exists even at thermal equilibrium and not only in the nonequilibrium iso-configurational conditions investigated in Refs. 1, 2, 3, 44. Therefore, we conclude that equilibrium concepts can be used to understand its origin and we suggest that the mechanism behind the behaviour in Fig. 2 is quite generic and not specifically due to an underlying nonequilibrium jamming transition at $T=0$. Indeed, we find in the literature several observations of similar anomalies observed in equilibrium conditions in systems of soft repulsive particles, for instance for Hertzian spheres [16] or in the Gaussian core model [17, 18]. To our knowledge, the close correspondence between nonequilibrium observations near the jamming transition, and these earlier reports at thermal equilibrium was not noted before.

Physically, the anomaly is a direct consequence of particle softness [16, 17, 18, 19]. The system behaves as 


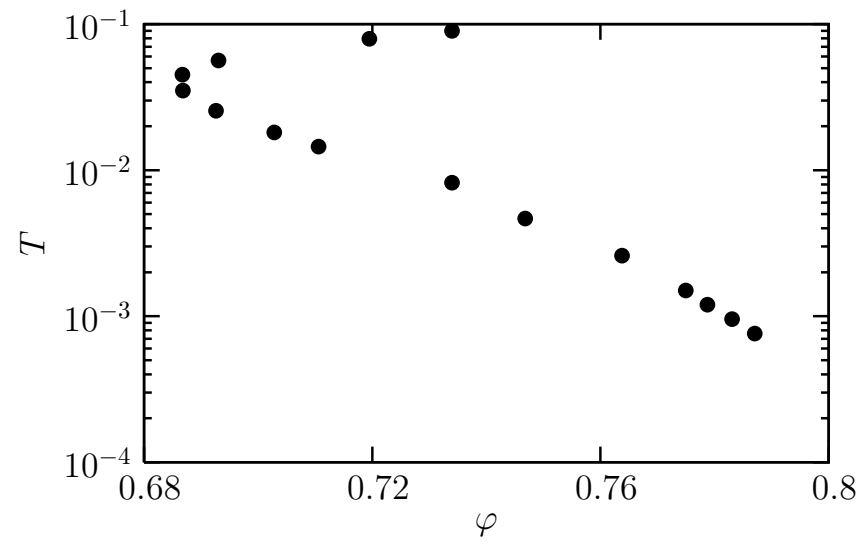

FIG. 3: Location of the maximum of the first peak of $g(r)$ in a temperature $(T)$ - volume fraction $(\varphi)$ phase diagram. This location is itself a nonmonotonic function of temperature. In particular in the low $T$ regime, it shifts to large $\varphi$ when $T$ decreases, as the system is able to find more efficient ways to pack non-overlapping particles.

an effective hard sphere fluid at sufficiently low volume fraction where it can easily minimize energy by avoiding particle overlaps. In this regime, the height of the peak increases with $\varphi$, just as it does in a hard sphere fluid. When the volume fraction increases, however, it becomes harder, and thus entropically less favourable, to avoid particle overlaps as the system must find more efficient ways to pack the particles. Thus, the system may gain free energy by increasing the disorder at the expense of allowing particle overlaps, which are energetically not very costly for soft repulsions such as the one in Eq. (11). In this regime, disorder increases when increasing $\varphi$, and this broadens the first peak of $g(r)$ and decreases its height. We note that similar competitions are invoked to explain an even larger set of anomalous thermodynamic behaviours, as observed for instance in widely studied network-forming liquids such as silica 20] and water 21, 22].

Since we attribute the anomaly to a competition between energy and entropy, the outcome of this competition can be expected to have an interesting evolution with temperature. Thus, we now turn to the influence of temperature on the nonmonotonic behaviour described in Fig. 2. In Fig. 3, we follow the locus of the maximum of $g(r)$ in a $(T, \varphi)$ phase diagram, as predicted from the HNC approximation. This figure confirms that equilibrium theory predicts a fairly nontrivial temperature evolution of the anomaly location, which is itself a nonmonotonic function of the temperature, with a crossover near $T \approx 0.04$. For $T>0.04$ the anomaly shifts to larger density for increasing temperature, while the opposite behaviour is found for $T<0.04$.

The low temperature behaviour, $T<0.04$, is readily understood on the basis of the equilibrium argument laid out above. When $T$ decreases, the entropic penalty to find efficient packing of the particles contributes less to the free energy, and it is preferable to avoid the energetically costly particle overlaps up to larger volume fractions. Thus, from equilibrium considerations one would naturally predict qualitatively the low temperature behaviour reported in Fig. 3. It reflects the physical intuition that thermal annealing should help the system to 'solve the packing problem' more efficiently.

Temperature has a second, competing effect which explains the high temperature behaviour in Fig. 3. When $T$ gets larger, particles acquire more kinetic energy, and they can more easily overlap. Thus the 'effective' particle diameter gets reduced by a simple thermal effect when $T$ increases [1, 9], and this effect must be compensated by an increase of the volume fraction. Therefore, temperature has two opposite effects which combine to yield the behaviour reported in Fig. 3, and explain the crossover near $T \approx 0.04$.

What happens if temperature is decreased further? We mentioned in the introduction that our equilibrium calculations eventually become inapplicable since a glass transition should take place when the structural relaxation time gets very large. In a simulation or an experiment, there will be a temperature below which the system will eventually be frozen into some amorphous configuration [9]. It is possible to describe this glass physics using liquid state theory, but this requires considerably more advanced techniques [8, 23, 24, 25].

Still, a number of conclusions naturally follow from the above equilibrium results. First, at low but finite temperatures, the system is deep into the glass region. Although it cannot relax to equilibrium on accessible timescales, it attempts to do so, albeit at a very slow pace. Thus, the physical explanation given above for the very existence of the structural anomaly should still apply in this regime, which is the one explored in Refs. [1, 4]. The structural anomaly should thus be 'frozen in' by the glass transition, which involves no important change of the structure. Second, we have shown above that the location of the maximum is ruled by two competing effects. In the glass phase, only the second one remains effective, as the system is not able to explore different configurations efficiently and find better particle packings. Thus, the evolution of the anomaly at low temperature should change again as the glass transition is crossed, and revert to the high temperature behaviour of Fig. 3 . Overall, thus, we predict that the locus of the structural anomaly displays three regimes due to the competing effects of (i) thermal fluctuations reducing the effective particle diameter, (ii) the entropy / energy competition favouring more efficient packing at low temperatures, (iii) the intervening glass transition. In a $(T, \varphi)$ phase diagram, the data should thus follow a nontrivial ' $\mathcal{S}$-shape'. This theoretical prediction is our main new result.

We confront these theoretical considerations to numer-

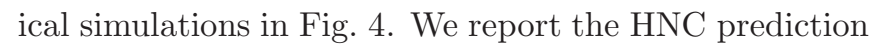




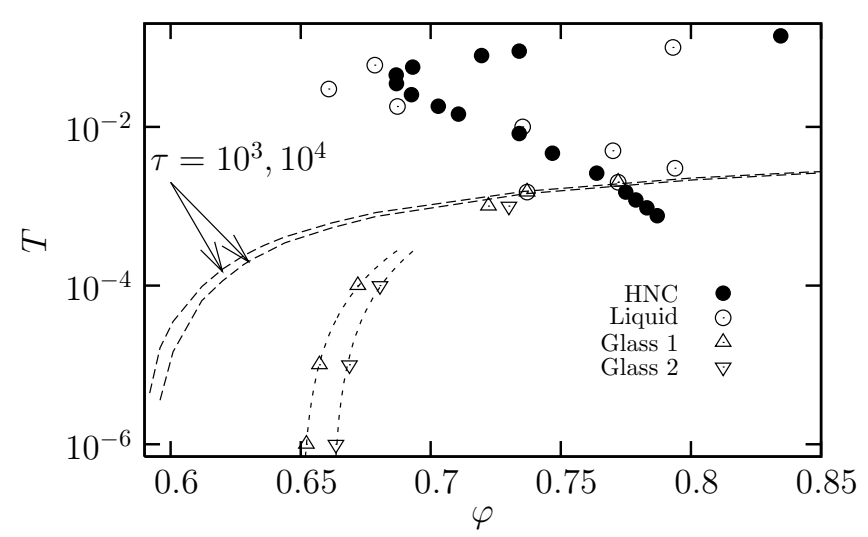

FIG. 4: Location of the maximum of the first peak of $g(r)$ in a temperature volume fraction phase diagram from HNC (filled circles), equilibrium simulations (open circles), nonequilibrium glass configurations (open triangles). The glass transition are defined as iso-relaxation timescales lines, with two different large values, from Ref. 9]. The location of the maximum follows a nontrivial $\mathcal{S}$-shape, as predicted theoretically.

for the location of the structural anomaly from Fig. 3 and compare it to the outcome of molecular dynamics simulations at thermal equilibrium. To obtain these data points, we made use of the extensive set of data of Ref. [9], where a 50:50 binary mixture of harmonic spheres with size ratio 1.4 was studied using molecular dynamics. A mixture is needed in the simulation to avoid crystallization. When necessary, we performed additional simulations to explore a broader range of state points, using the same techniques. We use a large system size, $N=8000$, where finite size effects are negliglible both at equilibrium [9] and at very low temperatures [7, 12].

We find a very good qualitative agreement between the equilibrium behaviour predicted using $\mathrm{HNC}$ and the outcome of computer simulations at sufficiently high temperatures, $T>0.003$, confirming that HNC performs quite well for harmonic spheres in this range of densities and temperatures. The nonmonotonic temperature behaviour and the location of the crossover near $T \approx 0.04$ are well reproduced. Quantitative agreement cannot be expected, as size polydispersity was not taken into account in our theoretical calculations.

We then report glass transition lines, defined from the temperature where the structural relaxation time, $\tau$, becomes larger than some prescribed value. We report two lines corresponding to two large values, $\tau=10^{3}$ and $\tau=10^{4}$, in the reduced time units used in molecular dynamics simulations [9]. It is very difficult to maintain thermal equilibrium below these lines in computer simulations. These glass lines meet the locus of structural anomaly near $\varphi \approx 0.8$ and $T \approx 0.0025$, which shows that the maximum of $g(r)$ cannot be observed in equilibrium conditions in computer simulations below $T=0.0025$. This also suggests that both phenomena are not directly connected.

At even lower temperatures, it becomes ambiguous to locate a unique maximum of $g(r)$, as this should in principle depend on the way the glass configurations have been prepared. To illustrate this difficulty, and therefore the nonequilibrium nature of the low temperature behaviour, we numerically prepared two glasses of harmonic spheres: 'Glass 1' is very rapidly quenched from a high temperature to well below the glass transition, while 'Glass 2' is very slowly annealed across $T_{g}$. For both glasses, we track the location of the $g(r)$ maxima down to very low temperatures, see Fig. 4. As discussed above, these data confirm the behaviour predicted at low temperature when no structural relaxation can occur. The location of the maximum shifts to lower volume fraction when temperature decreases. This is precisely the regime of temperature described in previous work [1, 4], and our results for 'Glass 1' agree with those earlier reports. In Fig. 4. we report the low temperature scaling behaviour deduced from considering the effect of thermal fluctuations alone, $T \sim\left(\varphi-\varphi_{c}\right)^{2}$, where the exponent ' 2 ' is the same as in the pair potential (10) and $\varphi_{c}$ denotes the location of the jamming transitions in the $T=0$ limit. These lines separate in the glass phase a region where the glass is dominated by repulsion at low volume fraction, from a region where particles are compressed at large volume fraction, and the system resembles a compressed emulsion.

The nonequilibrium nature of the jamming transition is illustrated from the difference between our two glasses in Fig. 4. They both display a line of structural anomaly, but these two lines are distinct and define jamming transitions at distinct densities, $\varphi_{c}=0.65$ and $\varphi_{c}=0.662$. The dependence of $\varphi_{c}$ on the preparation protocol in the $T=0$ limit has been discussed before [10, 11, 12, 13]. The data in Fig. 4 show that these considerations apply to finite temperatures as well, and to the structural anomaly in particular. Therefore, this figure illustrates the fact that nonequilibrium jamming transition lines are as ambiguously defined as glass transition lines, since they both strongly depend upon operational definitions [12, 26, 27]. These two concepts can only become protocol-independent in theoretical calculations [8, 25].

The crossover between the very low temperatures investigated in previous work, and the equilibrium regime studied here is also interesting. In the regime $T \approx$ $0.001-0.003$, the system is an aging glass at large density, but a fluid at low density. For the fluid state points the first peak of $g(r)$ increases with $\varphi$ and the extrapolated maximum is at very large $\varphi$. For the aging glasses, however, the peak decreases with $\varphi$ as the glass maximum should follow the low temperature trend. Thus, these two tendencies produce a maximum when the glass transition is crossed, and the glass and jamming lines in Fig. 田'kiss' in this temperature regime. Of course, the exact location of the maximum depends on the aging time. This effect 
was not reported before [1, 4], as the structure does not age very much at the very low temperatures studied in these earlier reports, $T / T_{g} \approx 1 / 1000-1 / 10$.

It is interesting to remark the close similarity between the effect of glass annealing in nonequilibrium situations, and the effect of decreasing the temperature at equilibrium: they both shift the maximum of $g(r)$ to larger $\varphi$ for the same physical reason. This correspondence emphasizes once more that the physics of the structural anomaly described in this paper is in fact deeply rooted in equilibrium concepts. The $T \rightarrow 0$ limit is no exception, but it makes the distinction between repulsive and compressed regimes sharper, as the energy of the ground state of the system is either 0 or positive [7].

To conclude, we have used a simple equilibrium theoretical approach to study the existence and location of a structural anomaly reported in recent experiments in soft repulsive spheres in nonequilibrium conditions. We have suggested that the structural anomaly reported in these articles can be explained using purely equilibrium concepts, and several aspects of its behaviour are indeed well described using liquid state theory. The very existence of an anomaly relies on a standard competition between entropy and energy in the free energy of these very soft particles, and we have suggested that the anomaloy belongs to a much broader class of similar anomalies studied in the literature. In the present case, it directly results from the extreme softness of particles described by pair potentials such as Eq. (11).

For standard models describing atomic liquids, such as Lennard-Jones potentials, the repulsive core is much steeper, and they are thus governed by a different physics upon compression. Indeed, the large density limit of both types of systems is very different, as the harmonic spheres behave as an ideal gas in this limit [17. In the context of glassy physics, a recent quantitative comparison between harmonic spheres and Lennard-Jones models of the glass transition has also confirmed the different nature of their glassy dynamics and of its evolution with density [28].

Additionally, we have discussed three competing effects of the temperature which control the location of the anomaly in the phase diagram, and predicted a nontrivial ' $\mathcal{S}$-shape', confirmed by numerical simulations. We have finally shown that the anomalous structural behaviour of soft particles discussed in Refs. [1, 2, 3, 4] in fact occurs over a broad, continuous range of densities and temperatures, as it is directly affected by sample preparation effects.

In future work 25], we plan to extend the present liquid state calculations to describe analytically the properties of dense systems of soft particles down to very low temperatures, where ergodicity breaking must explicitely be taken into account.

We thank G. Biroli, L. Cipelletti, W. Kob, G. Szamel, and F. Zamponi for useful feedback and construc- tive criticisms about this work. We thank X. Cheng for providing his experimental data from Ref. [3]. H. Jacquin acknowledges financial support from Capital Fund Management (CFM) Foundation, and from the LCVN in the early stages of this work. L. Berthier is partially supported by the ANR Dynhet.

[1] Z. Zhang, N. Xu, D. T. N. Chen, P. Yunker, A. M. Alsayed, K. B. Aptowicz, P. Habdas, A. J. Liu, S. R. Nagel, and A. G. Yodh, Nature 459, 230 (2009).

[2] X. Cheng, arXiv:0905.2788 (2009).

[3] X. Cheng, arXiv:0911.1943 (2009).

[4] N. Xu, arXiv:0911.1576 (2009).

[5] A. Donev, S. Torquato, and F. H. Stillinger, Phys. Rev. E 71, 011105 (2005).

[6] L. E. Silbert, A. J. Liu, and S. R. Nagel, Phys. Rev. E 73, 041304 (2006).

[7] C. S. O'Hern, S. A. Langer, A. J. Liu, and S. R. Nagel, Phys. Rev. E 68, 011306 (2003).

[8] G. Parisi and F. Zamponi, Rev. Mod. Phys. (in press); arXiv:0802.2180

[9] L. Berthier and T. A. Witten, EPL 86, 10001 (2009); Phys. Rev. E 80021502 (2009).

[10] A. Donev, S. Torquato, F. H. Stillinger, and R. Connelly, Phys. Rev. E 70, 043301 (2004).

[11] A. Donev, F. H. Stillinger, and S. Torquato, J. Chem. Phys. 127, 124509 (2007).

[12] P. Chaudhuri, L. Berthier, and S. Sastry, arXiv:0910.0364 (2009).

[13] M. Hermes and M. Dijkstra, arXiv:0903.4075.

[14] J. P. Hansen and I. R. McDonald, Theory of Simple Liquids (Elsevier, Amsterdam, 1986).

[15] L. Berthier, E. Flenner, H. Jacquin, and G. Szamel, arXiv:0912.1738

[16] J. C. Pamies, A. Cacciuto, and D. Frenkel, J. Chem. Phys. 131, 044514 (2009).

[17] A. Lang, C. N. Likos, M. Watzlawek, and H. Löwen, J. Phys.: Condens. Matter 12, 5087 (2000).

[18] W. P. Krekelberg, T. Kumar, J. Mittal, J. R. Errington, and T. M. Truskett, Phys. Rev. E 79, 031203 (2009).

[19] A. A. Louis, P. G. Bolhuis, and J. P. Hansen, Phys. Rev. E 62, 7961 (2000).

[20] M. S. Shell, P. G. Debenedetti, and A. Z. Panagiotopoulos, Phys. Rev. E 66, 011202 (2002).

[21] J. R. Errington and P. G. Debenedetti, Nature 409, 318 (2001).

[22] W. P. Krekelberg, J. Mittal, V. Ganesan, and T. M. Truskett, Phys. Rev. E 77, 041201 (2008).

[23] R. Monasson, Phys. Rev. Lett. 75, 2847 (1995).

[24] M. Mézard and G. Parisi, Phys. Rev. Lett. 82, 747 (1999).

[25] H. Jacquin et al. (in preparation).

[26] F. Krzakala and J. Kurchan, Phys. Rev. E 76, 021122 (2007).

[27] S. Torquato, T. M. Truskett, and P. G. Debenedetti, Phys. Rev. Lett. 84, 2064 (2000).

[28] L. Berthier and G. Tarjus, Phys. Rev. Lett. 103, 170601 (2009). 